\title{
Transplantation of vascular endothelial growth factor 165-transfected endothelial progenitor cells for the treatment of limb ischemia
}

\author{
SHENG WANG, ZHONG CHEN, XIAOBIN TANG, HUI LIU, LIAO YANG and YANYANG WANG \\ Department of Vascular Surgery, Beijing Anzhen Hospital, Capital Medical University, Beijing 100029, P.R. China
}

Received October 9, 2014; Accepted June 22, 2015

DOI: $10.3892 / \mathrm{mmr} .2015 .4100$

\begin{abstract}
The present study aimed to investigate the effects of neovascularization in rabbits with limb ischemia transplanted with vascular endothelial growth factor (VEGF)165-transfected endothelial progenitor cells (EPC). Bone marrow mononuclear cells were isolated by gradient centrifugation, cultured in M199 culture medium and induced into EPCs using VEGF, basic fibroblast growth factor, and insulin-like growth factor-1, and subsequently identified. The EPCs were transfected with Adv-green fluorescent protein-VEGF165 and the proliferation potential of the cells was determined using an MTT assay. The protein expression levels of VEGF were measured by detecting its concentration levels in the supernatant using an ABC-ELISA assay. A rabbit hind limb ischemic model was established and randomly divided into three groups: (A) Control group, (B) EPC-transplanted group, and (C) Ad-VEGF165/EPCs-transplanted group. The effects of transplantation and the levels of recanalization were detected. Incorporation of the transplanted cells into the ischemic region was confirmed by 5-bromodeoxyuridine staining, and the levels of recanalization were measured by computer tomography ateriography and immunohistochemical staining. Bone marrow-derived EPCs were induced, cultivated, and successfully identified. The results of the present study determined the optimum transfection ratio that promoted the growth of EPCs. The EPCs were successfully transfected with VEGF165, and EPC proliferation was not affected by the transfection. The supernatant protein concentration levels of VEGF were markedly higher in the VEGF165-transfected group, as compared with those of the control group. Introduction of the transplanted cells into the ischemic region of group C occurred more efficiently, as compared with groups A and B. The recanalization capillary density in group $\mathrm{C}$ was significantly higher, as compared with groups A and B. VEGF gene transfection was able to improve the quality of EPCs, and the
\end{abstract}

Correspondence to: Dr Zhong Chen, Department of Vascular Surgery, Beijing Anzhen Hospital, Capital Medical University, 2 Anzhen Road, Beijing 100029, P.R. China

E-mail: xgtg188@163.com

Key words: transplantation, genes, stem cells, ischemia response of rabbits with limb ischemia to transplantation with VEGF-transfected EPCs was significantly better, as compared with transplantation with EPCs alone.

\section{Introduction}

Limb ischemia is deleterious to human health, and many patients are not suitable for surgical treatment due to various factors, including anatomy. Therefore, the prognosis of limb ischemia is poor, and causes great discomfort. In addition, therapeutic strategies are rarely successful, leading to limb amputation in many patients, specifically those who suffer from severe pain. Increasing the levels of angiogenesis and improving circulation in ischemia limbs may provide the most effective treatment, and is thus the subject of numerous studies $(1,2)$. Stem cell transplantation in the treatment of ischemic diseases has recently been investigated by numerous studies $(3,4)$, with animal model experiments and clinical studies being carried out in order to elucidate the effects of stem cell transplantation on ischemic diseases.

Stem cell transplantation has limitations, predominantly associated with the quality and quantity of endothelial progenitor cells (EPCs), which requires improvement in order for treatment to be successful. Using genetic engineering to improve the quality of EPCs, thus reducing the dosage of EPCs required for transplantation, may provide a promising method of limb ischemia treatment.

Vascular endothelial growth factor (VEGF) is an endothelial cell-specific growth factor and a regulator of physiological and pathological angiogenesis. In addition, VEGF is able to increase the permeability of capillary vessels to different macromolecules. VEGF consists of a family of polypeptide isoforms generated from a single-copy gene by alternative splicing of the primary transcript. VEGF is secreted by intact cells as a dimer of the four isoforms of 121, 165, 189, and 206 amino acids and are secreted from cells via a poorly characterized pathway (5). In the present study, in vitro EPCs were obtained from the differentiation of bone marrow mononuclear cells (BM-MNCs) extracted from New Zealand rabbits. VEGF165 gene transfection was subsequently performed, and the cells were transplanted into New Zealand rabbits with ischemic hind limbs. The levels of neovascularization and the effects of transplantation on limb ischemia were detected. The present study aimed to provide an experimental basis for the clinical application of stem cells and gene therapy in the treatment of ischemic diseases. 


\section{Materials and methods}

Animals. New Zealand rabbits (age, 3-4 months) were obtained from the Animal Laboratory of Beijing Institute of Heart Lung and Blood Vessel Diseases (Beijing, China). The animals were maintained at a room temperature of $20-22^{\circ} \mathrm{C}$ and humidity of $40-70 \%$ under a 12-h light/dark cycle, with access to food and water ad libitum. The study was approved by the ethics committee of Beijing Anzhen Hospital, Capital Medical University (Beijing, China).

EPC isolation, culture, and identification. New Zealand rabbits were intravenously injected via the marginal ear vein with $3 \%$ sodium pentobarbital $(30 \mathrm{mg} / \mathrm{kg}$; Beijing Tianlai Biologic Products, Inc., Beijing, China), and bilateral iliac crest multipoint punctures were performed prior to marrow fluid aspiration. The quantity of bone marrow obtained from a single puncture was $\sim 10-15 \mathrm{ml}$. Gradient centrifugation was performed in Ficoll (Sigma-Aldrich, St. Louis, MO, USA) in order to separate the mononuclear cells. The cells were then plated onto culture dishes in M199 culture medium (Gibco Life Technologies, Carlsbad, CA, USA) supplemented with 15\% fetal bovine serum (GE Healthcare Life Sciences, Logan, UT, USA), $20 \mu \mathrm{g} / \mathrm{ml}$ VEGF (PeproTech EC Ltd., London, UK), $2 \mu \mathrm{g} / \mathrm{ml}$ alkaline fiber cell growth factor (bFGF; PeproTech EC Ltd.), $2 \mu \mathrm{g} / \mathrm{ml}$ insulin-like growth factor-1 (IGF-1; PeproTech EC Ltd.), and half of the medium was changed every 3-4 days.

Following 10 days of culture, the cells were collected and fixed in sections, prior to being observed under a transmission electron microscope (TEM; JEM-1010; JEOL., Ltd., Tokyo, Japan). The cells were fixed and mounted onto slides following 14 days of culture, and indirect immunofluorescence was carried out using the following primary antibodies: Rabbit-anti-von Willebrand factor (vWF) polyclonal antibody (sc14014; Santa Cruz Biotechnology, Inc., Dallas, TX, USA), and rabbit-anti-CD133 monoclonal antibody (ab19898; Abcam plc, Cambridge, UK), and the following secondary antibodies: Fluorescein isothiocyanate (FITC)-labeled goat-anti-rabbit antibody (sc3839; Santa Cruz Biotechnology, Inc.), and tetramethylrhodamine (TRITC)-labeled goat-anti-rabbit antibody (sc3841; Santa Cruz Biotechnology, Inc.). All dilutions were $1: 200$.

In vitro gene transfer in EPCs. Following 14 days in culture, the EPCs were transfected with a recombinant VEGF165-encoding gene (Ad-VEGF165) using Lipofectamine 200 (Invitrogen Life Technologies, Carlsbad, CA, USA), and post-transfection, the cells were incubated at $37^{\circ} \mathrm{C}$ in an atmosphere containing $5 \% \mathrm{CO}_{2}$ for $2 \mathrm{~h}$. Three multiplicities of infection (MOI; 10, 50, and 100) were used, and cellular proliferation was assessed by MTT assay (Sigma-Aldrich). A total of $20 \mathrm{ml}$ MTT solution $(5 \mathrm{mg} / \mathrm{ml})$ was added to each well, and colorimetric assays were conducted at $\lambda=570 \mathrm{~nm}$ following incubation for $4 \mathrm{~h}$. Following experimentation, the EPCs were transfected with 50 MOI Ad/VEGF165, and the transfection efficiency was measured by fluorescence using a bx51m fluorescence microscope (Olympus Optical Co., Ltd., Tokyo, Japan). EPCs were transfected with either Ad or Ad-VEGF165, and the proliferative activity of the non-transfected cells (non/EPCs), Ad-transfected EPCs (Ad/EPCs), and
Ad-VEGF165-transfected EPCs (Ad-VEGF/EPCs) was evaluated.

Analysis of the protein expression levels of VEGF by ELISA. On days 1, 4, 7, and 14 following gene transfer, the Ad-VEGF165/EPCs, Ad/EPCs, and non /EPCs were reseeded ( $5 \times 10^{3} / 200 \mu \mathrm{l} /$ well), and the supernatant was collected. The protein expression levels of VEGF were detected using an ABC-ELISA (Beijing Jingmei Biologic Products,Inc., Beijing, China) with the supernatant as the sample.

Establishment of a New Zealand rabbit model with hind limb ischemia. Sodium pentobarbital was intravenously injected into the marginal ear vein of male New Zealand rabbits, and a $2.5 \mathrm{~cm}$ longitudinal incision was made at the groin. The incision was made in the skin and subcutaneous tissue, and the soft tissues were subsequently separated from the femoral artery, which was carefully dissected from the femoral vein and nerve. Each branch of the femoral artery was then dissected, and the deep femoral arteries, superficial epigastric arteries, and inferior epigastric arteries were sectioned and ligated. During the surgical procedure, the femoral vein and nerve were protected.

EPC transplantation. Following culture for 10 days the EPCs were digested with trypsin (Gibco Life Technologies) in order to obtain a single cell suspension, and the cell concentration was adjusted to $5 \times 10^{5}$ cells $/ \mathrm{ml}$. Purebred male New Zealand rabbits were randomly divided into three groups $(n=20)$ two days following the ischemic surgical procedure: (A) A control group that received culture medium; a (B) Ad/EPCs-transplantation group; and a (C) Ad-VEGF165/EPCs-transplantation group. A total of 10 points $(0.5 \times 0.5 \mathrm{~cm})$ were selected in each $\mathrm{New}$ Zealand rabbit ischemia limb for injection of the cell suspension, and $0.5 \mathrm{ml}$ cell suspension $\left(5 \times 10^{6}\right)$ being injected into each point $(5 \mathrm{ml} / \mathrm{rabbit}$ in total). The group A rabbits were injected with $5 \mathrm{ml} \mathrm{M} 199$ medium.

Monitoring of transplanted cells. In order to monitor the fate of the transplanted EPCs, the EPCs were labeled with 5-bromodeoxyuridine (Brdu; Sigma-Aldrich). Two New Zealand rabbits from each group were transplanted with Brdu-EPCs, as previously described (6). Seven days post-transplantation, the rabbits were sacrificed via the injection of $3 \%$ sodium pentobarbital, and the gastrocnemius muscle of the ischemic limbs was obtained and sectioned $(6 \mu \mathrm{m})$ prior to being frozen and assayed by Brdu immunohistochemical staining.

Histological assessment. On days 7, 14, 21, and 28 following the surgical procedure, two rabbits from each group were randomly selected and sacrificed via the injection of $3 \%$ sodium pentobarbital, and the gastrocnemius muscle of the ischemia limbs was obtained and sectioned prior to being fixed in paraffin. The tissue specimens were examined by hematoxylin and eosin (HE) staining, and the endothelial cells in the tissue samples were determined by immunohistochemical staining using a vWF antibody. The capillary density of the vWF-positive cells was determined by counting the number of cells in five random high-power (magnification, $\mathrm{x} 200$ ) fields using a CKX-31 microscope (Olympus Optical Co., Ltd.). 
Monitoring of the skin temperature changes of New Zealand rabbits following transplantation. On days 2, 4, 7, 14, and 28 following transplantation, changes in skin temperature were monitored by scanning the lower limbs of the rabbits using an infrared scanner (FLIR Systems, Wilsonville, OR, USA).

Analysis of the levels of recanalization in the New Zealand rabbits by computer tomography ateriography (CTA). On the 14th day following transplantation, the New Zealand rabbits of each group underwent CTA using a 64-slice spiral computerized tomography scanner (Siemens, Munich, Germany). The rabbits were anesthetized by intravenous injection of $3 \%$ sodium pentobarbital into the marginal ear vein, and a scalp needle was fixed following anesthesia to connect the syringes. The CT scans were carried out, and the lower limb artery reconstruction of each group was examined.

Statistical analysis. All data were analyzed by SPSS 11.5 (SPSS, Inc., Chicago, IL, USA). The results of the groups were compared using a one-way analysis of variance, and R-E-G-W-Q and Tukey tests. $\mathrm{P}<0.05$ was considered to indicate a statistically significant difference.

\section{Results}

EPC isolation, culture, and identification. A small number of primary cells adhered to the surface of the culture vessels following differentiation and 48-72 h culture. The number of adhering cells gradually increased, and formed spindles (Fig. 1A and B). Following eight days of culture, numerous spindles were observed, having formed by the connection of adherent cells growing in a straight line (Fig. 1C). These observations are characteristic of endothelial cells. Numerous cell masses appeared following 7-10 days culture, and the spindle cells formed a bud at the edge of the cell masses, this structure resembled a blood island. Following culture for $\sim 14$ days, the cells fused together forming large funicular structures (Fig. 1D).

Nuclei, mitochondria, and vacuoles were observed in the cells using TEM, along with a few pinocytotic vesicles, which were characteristic of endothelial cell structure. Microvilli were visible close to the cellular membrane (Fig. 2A). Using anti-vWF polyclonal antibody and FITC-labeled antibody as primary and secondary antibodies, respectively, the spindle cells emitted green fluorescence under the fluorescence microscope (Fig. 2B). Using anti-CD133 monoclonal antibody and TRITC-labeled antibody as primary and secondary antibodies, respectively, the spindle cells emitted red fluorescence under the fluorescence microscope (Fig. 2C). Since vWF and CD133 are components of endothelial cells, these results indicate that the BM-MNCs had successfully differentiated into EPCs.

Monitoring of transplanted cells. The Brdu-labeled EPCs emitted green fluorescence, as determined by fluorescence microscopy (Fig. 3).

Proliferative activity of the transfected EPCs. An MTT assay was used to detect the proliferative activity of the transfected EPCs. Three multiplicities of infection (MOI: 10, 50, and 100) were investigated (Fig. 4A). Following experimentation, the EPCs were transfected with 50 MOI Ad/VEGF165, and the efficiency of transfection was measured by fluorescence microscopy. The EPCs transfected with Adv-GFP-VEGF165 contained a green fluorescent protein gene, and $24 \mathrm{~h}$ post-transfection the majority of cells emitted green fluorescence, as determined by fluorescence microscopy (Fig. 4B). The EPCs were transfected with either Ad or Ad-VEGF165, and the proliferative activity of the non/EPCs, Ad/EPCs, and Ad-VEGF/EPCs were evaluated. The proliferative activities of the non/EPCs, Ad/EPCs, and Ad-VEGF/EPCs were similar (Fig. 4C).

Protein expression levels of VEGF post-transfection. On the 1, 4, 7, 14 days following gene transfer, the Ad-VEGF165/EPCs, $\mathrm{Ad} / \mathrm{EPCs}$, and non/EPCs were reseeded, and the supernatant was collected. The protein expression levels of VEGF were detected in the supernatant using an ABC-ELISA. As shown in Fig. 5, the protein expression levels of VEGF in the Ad-VEGF165/EPCs were significantly higher, as compared with those of the non /EPCs $(\mathrm{P}<0.01)$ and Ad/EPCs $(\mathrm{P}<0.01)$ on the same day.

The limb ischemic model and survival of the transplanted cells. A total of $24 \mathrm{~h}$ following ischemic experimentation, the effects of ischemia were examined by CTA. As shown in Fig. 6A, distal artery occlusion was observed in the ischemic limb. Two New Zealand rabbits in each group were transplanted with Brdu-labeled EPCs, as previously described. On the 7 th day following transplantation, the gastrocnemius muscle of the ischemic limbs was sectioned and frozen, prior to being assayed by Brdu-immunohistochemical staining. Brdu-positive cells (brown) were observed (Fig. 6B), indicating the survival of transplanted EPCs and directional integration of the cells into the ischemic area, as well as EPC-induced angiogenesis.

Histological examination of the rabbits following transplantation. The tissue specimens were examined by HE staining, and the endothelial cells in the tissues were examined by immunohistochemical staining using the vWF antibody. The capillary density of vWF-positive cells was determined by counting the number of cells in five random high-power (magnification, x200) fields. Quantification of the histological analysis results revealed that the density of blood vessels in group $\mathrm{C}$ was significantly higher, as compared with groups $\mathrm{A}$ $(\mathrm{P}<0.01)$ and $\mathrm{B}(\mathrm{P}<0.01)$. In addition, the density of blood vessels in group B was significantly higher, as compared with group A $(\mathrm{P}<0.01)$ (Fig. 7).

Skin temperature changes in New Zealand rabbits following transplantation. On the 2nd , 4th , 7th , 14th , and 28th days following transplantation, changes in skin temperature were monitored. The skin temperatures of groups B and C were significantly higher, as compared with group A at the 14th, and 28th days $(\mathrm{P}<0.01)$ (Fig. 8).

Analysis of recanalization of the New Zealand rabbits, as determined by CTA. On the 14th day following transplantation, New Zealand rabbits from each group underwent CTA using a Siemens 64-slice spiral CT. The lower limb artery reconstruction 
A
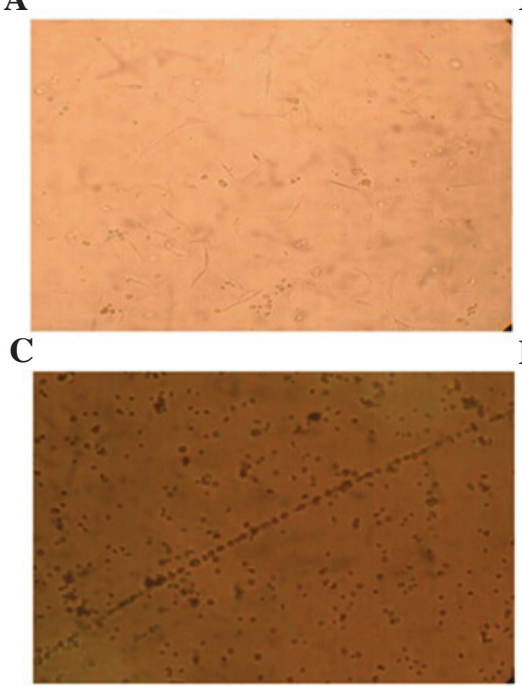

B
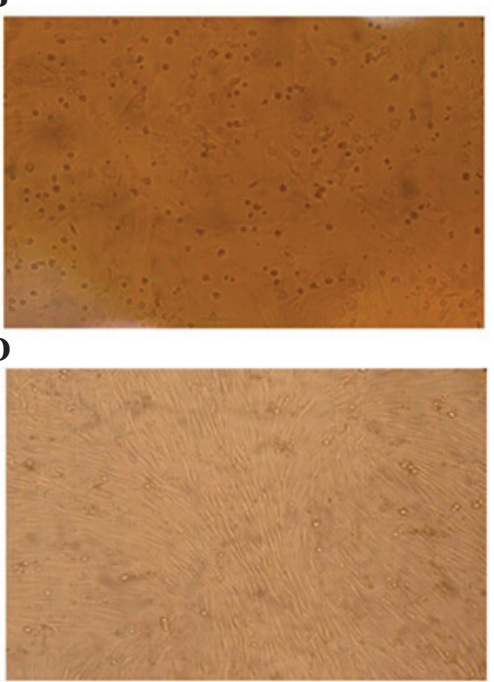

Figure 1. Culture of endothelial progenitor cells (EPCs). (A and B) EPCs formed spindles. Magnification, x20. (C) Numerous spindles were observed following 8 days of culture. (D) Following 14 days of culture, the EPCs fused together. Both magnification, $\mathrm{x} 10$.

A

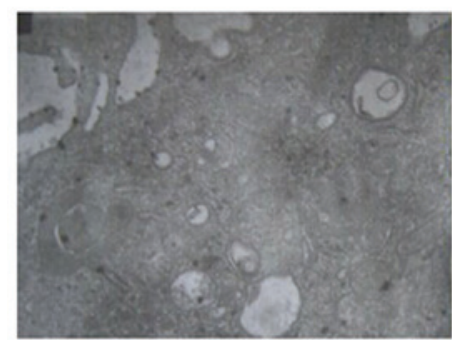

B

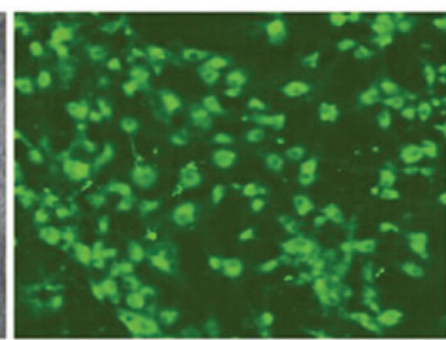

C

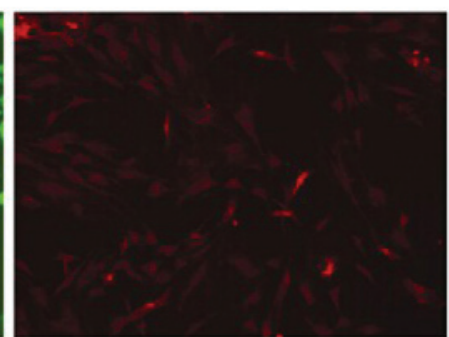

Figure 2. Identification of endothelial progenitor cells (EPCs). (A) A small number of pinocytotic vesicles could be observed in the EPCs. The cells stained positively for endothelial markers Magnification, x8,000. (B) Willebrand-factor (vWF) and (C) CD133, as determined by immunohistochemistry; magnification, x10.

of each group was examined. A higher number of small blood vessels in the ischemic area and greater collateral circulation was observed in group A, as compared with group B. The recanalization of group B was markedly higher, as compared with group A (Fig. 9). The establishment of collateral circulation of group $\mathrm{C}$ was observed following soft tissue removal (Fig. 10).

\section{Discussion}

Stem cells are non-specialized cells that have the ability to self-renew and differentiate into numerous cell lines. Stem cells are able to differentiate into various tissues and organs when stimulated by differentiation factors. EPCs have been the subject of numerous studies since their discovery, and their surface markers, mobilization, directional differentiation, and effects on angiogenesis in the treatment of cardiovascular diseases and cancers have been widely researched $(7,8)$. As compared with adult tissues, one of the advantages of stem cell transplantation is that they are easily modified, and may serve as gene therapy targets. Genetic modification of EPCs may improve their function, thus enhancing the effects of EPC transplantation.

Previous studies have reported that embryonic hematopoietic stem cells (HSCs) and EPCs originate from a common precursor cell, the blood-vascular stem cell (9-11). HSCs and

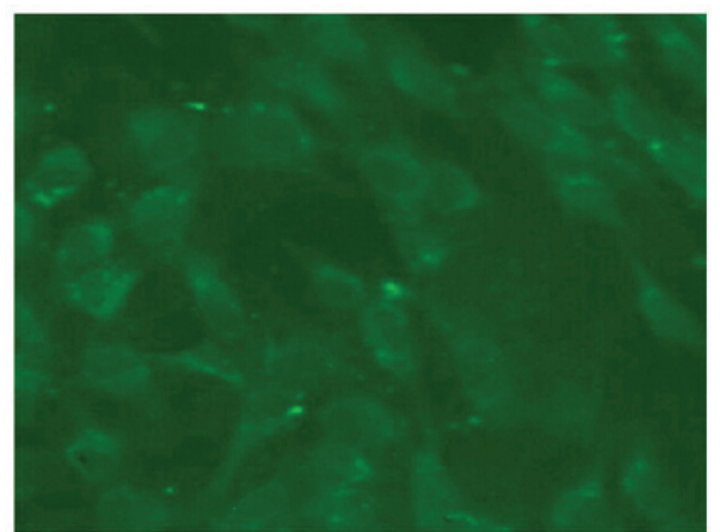

Figure 3. The 5-bromodeoxyuridine-labeled endothelial progenitor cells were observed by fluorescence microscopy, and the labeled cells emitted green fluorescence. Magnification, x40.

EPCs also share common antigens, including CD34, VEGF receptor 2, Tie-2, CD117, and stem cell antigen-1 (9,11). Since these initial reports on EPCs, this cell group has been further studied, and numerous methods of EPC differentiation have been reported (11-13).

The present study used a simple method to induce EPC differentiation. BM-MNCs were obtained via centrifugation 
A

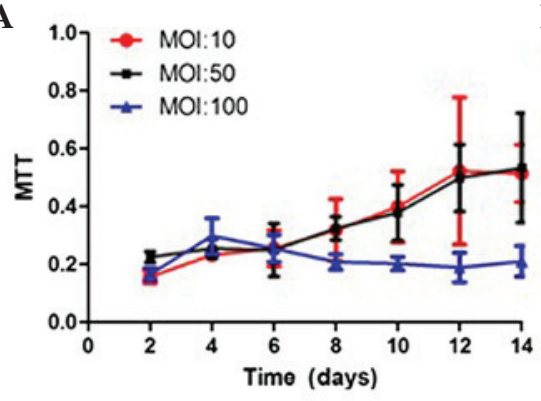

B

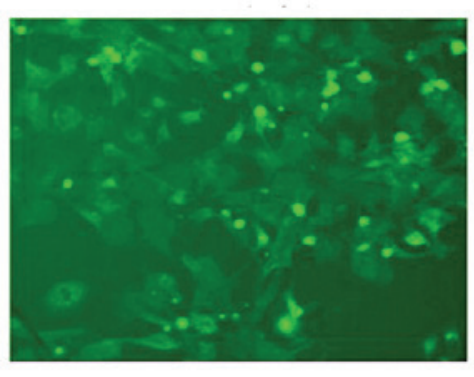

C

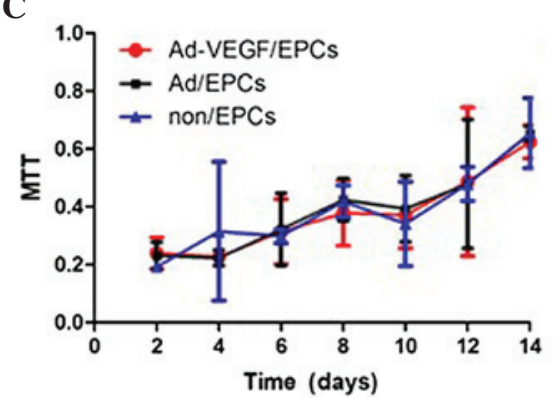

Figure 4. (A) Three different multiplicities of infection (MOI: 10, 50, and 100) were used. (B) The transfected endothelial progenitor cells (EPCs) emitted green fluorescence. Magnification, x10. (C) The proliferative activity levels of the non-transfected cells (non/EPCs), Ad-transfected EPCs (Ad/EPCs), and Ad-VEGF165-transfected EPCs (Ad-VEGF/EPCs) were similar. Data are presented as the mean \pm standard deviation.

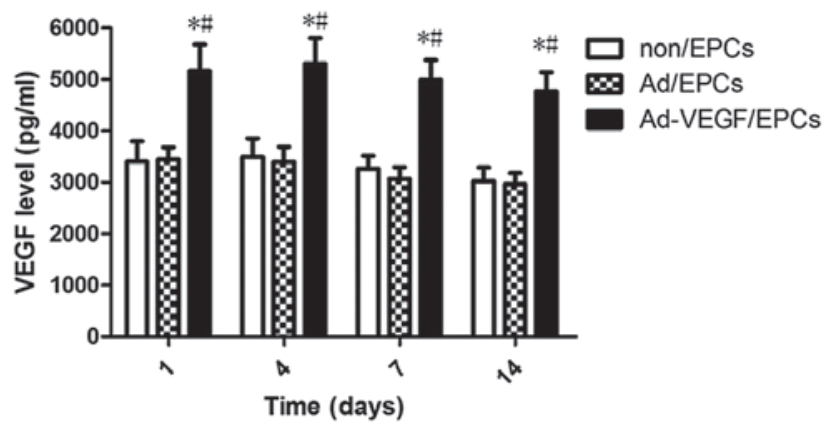

Figure 5. The protein expression levels of vascular endothelial growth factor (VEGF) in Ad-VEGF165-transfected EPCs (Ad-VEGF/EPCs) were higher, as compared with those of non-transfected cells (non/EPCs), and Ad-transfected EPCs (Ad/EPCs). ${ }^{*}<<0.01$, vs. the non/EPCs group; and ${ }^{\# P} \mathrm{P}<0.01$, vs. the $\mathrm{Ad} / \mathrm{EPCs}$ group. Data are presented as the mean \pm standard deviation.
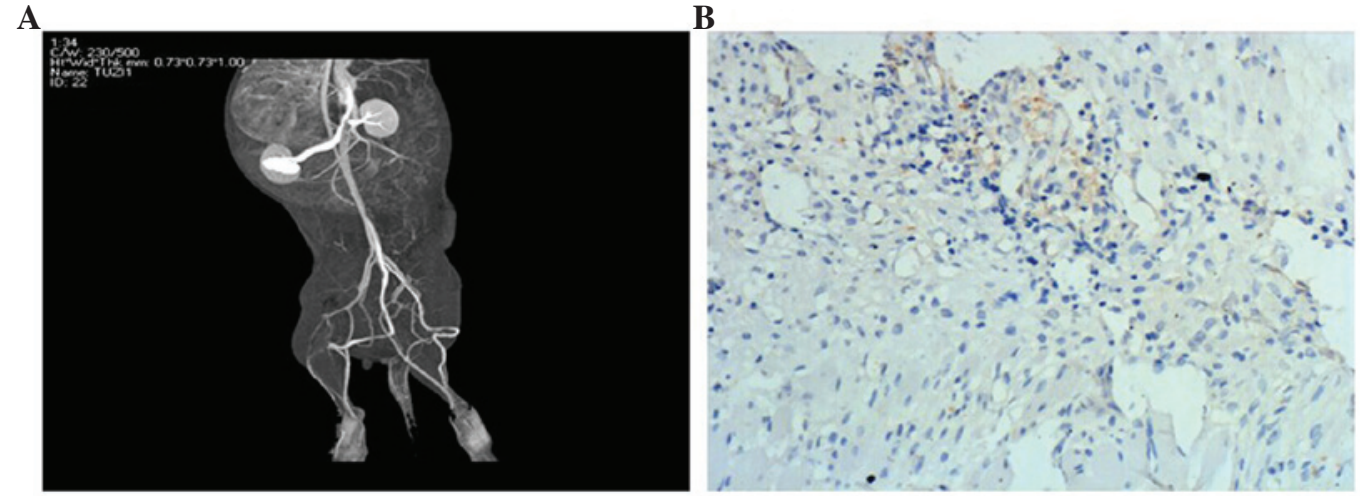

Figure 6. (A) Distal artery occlusion was observed in the ischemic limbs following ischemic experimentation. (B) Two New Zealand rabbits from each group were transplanted with 5-bromodeoxyuridine (Brdu)-labeled endothelial progenitor cells (EPCs), and the Brdu positive cells (brown) were observed. Magnification, $\mathrm{x} 200$.

using Ficoll centrifugal liquid, at a density of $1.0860 \pm 0.001$ BM-MNCs consist predominantly of lymphocytes and a small number of mononuclear cells and platelets, as well as other cells. The differentiation of BM-MNCs into EPCs was induced using VEGF, IGF-1, and bFGF.

During culture, the adherent cells formed numerous strips characteristic of EPCs, observations that have been reported in a previous study (14).

The presence of EPCs was verified by examining the presence of vWF, a specific surface maker of EPCs, and CD133, which is expressed on the surface of immature EPCs.
In the present study, EPCs were transfected with $50 \mathrm{MOI}$ Ad/VEGF165, since low MOI resulted in inefficient transfection, and a high MOI resulted in cellular damage. The results of the MTT assay demonstrated that cell proliferation was similar in the 10 and 50 MOI groups, however, in the 100 MOI group, cell growth arrest and fragments of necrotic cells were observed. Therefore, MOI 50 was used in the present study to ensure the transfection efficiency was as high as possible, and that the cells did not suffer damage. Satisfactory transfection efficiencies were achieved and the proliferative activities of Ad-GFP-VEGF165/EPCs, 
A
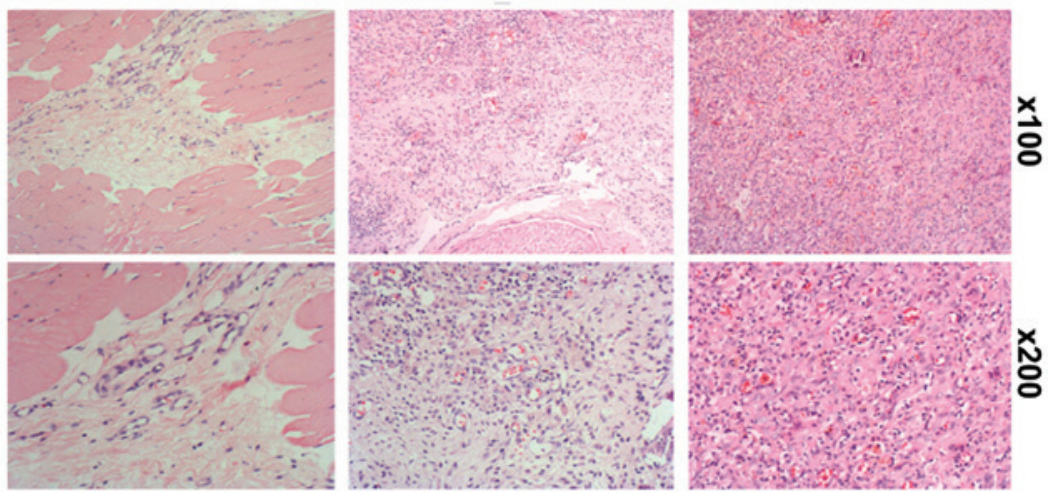

B

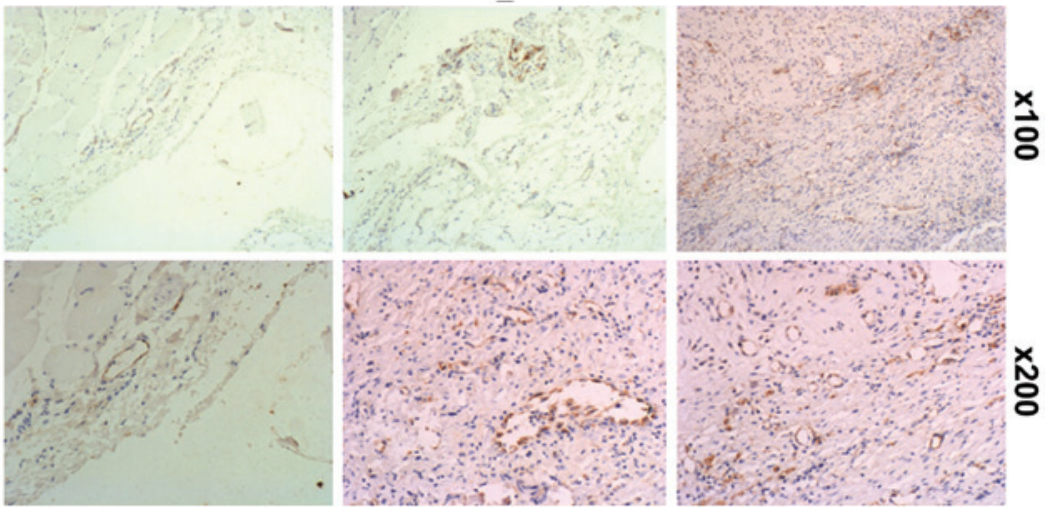

C

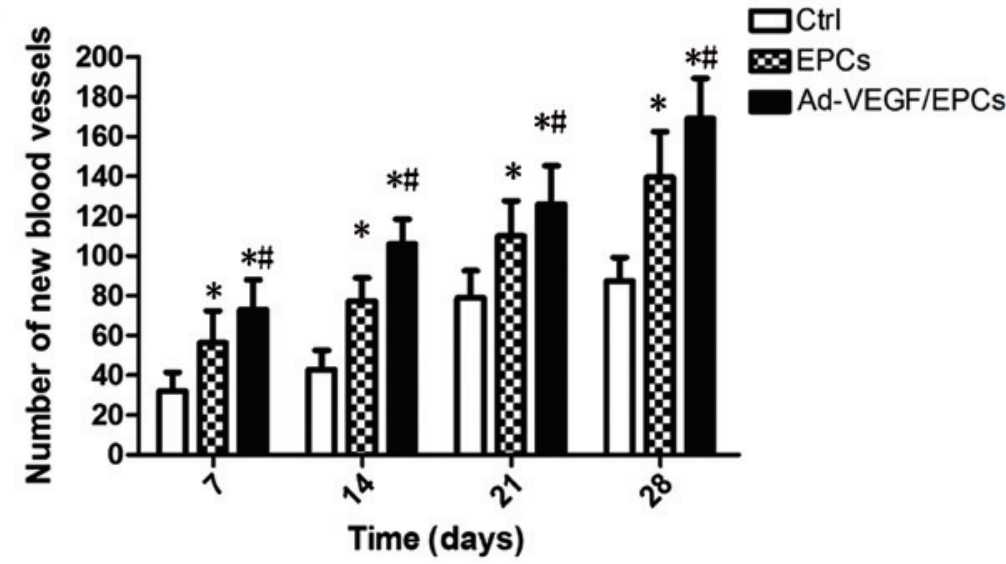

Figure 7. (A) Hematoxylin and eosin staining and (B) immunohistochemical staining using the von Willebrand-factor antibody. (C) The blood vessel densities were measured. " $\mathrm{P}<0.01$, vs. group $\mathrm{A}$; and ${ }^{\mathrm{H}} \mathrm{P}<0.01$, vs. group B. EPCs, endothelial progenitor cells; Ad-VEGF/EPCs, Ad-VEGF165-transfected EPCs.

Ad-GFP/EPCs, and EPCs non-Ad/EPCs were similar, which suggested that gene transfection with adenovirus had no effect on cell proliferation.

An ELISA assay was used to detect the concentrations levels of VEGF in the supernatant, demonstrating that the concentration levels of VEGF in the supernatant of Ad-GFP-VEGF165/EPCs was markedly higher, as compared with those of the Ad-GFP/EPCs and non-Ad/EPCs. EPCs possess a VEGF secretory function, and elevated concentration levels of VEGF in the supernatants of the cultured cells confirmed the presence of EPCs. The concentration levels of VEGF increased following gene transfection, which demonstrated the success of the transfection, and also provided evidence that VEGF has an important role following cell transplantation.

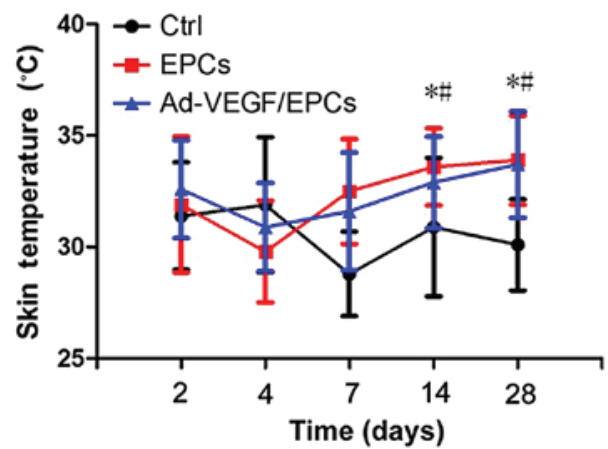

Figure 8. Skin temperature variation following transplantation. The skin temperatures of the rabbits of groups $\mathrm{B}$ and $\mathrm{C}$ were markedly higher, as compared with those of group A on the days 21 and 28 . ${ }^{*}<0.01$, group B, vs. group $\mathrm{A}$; and ${ }^{\#} \mathrm{P}<0.01$ group $\mathrm{C}$, vs. group $\mathrm{A}$. EPCs, endothelial progenitor cells; Ad-VEGF/EPCs, Ad-VEGF165-transfected EPCs. 

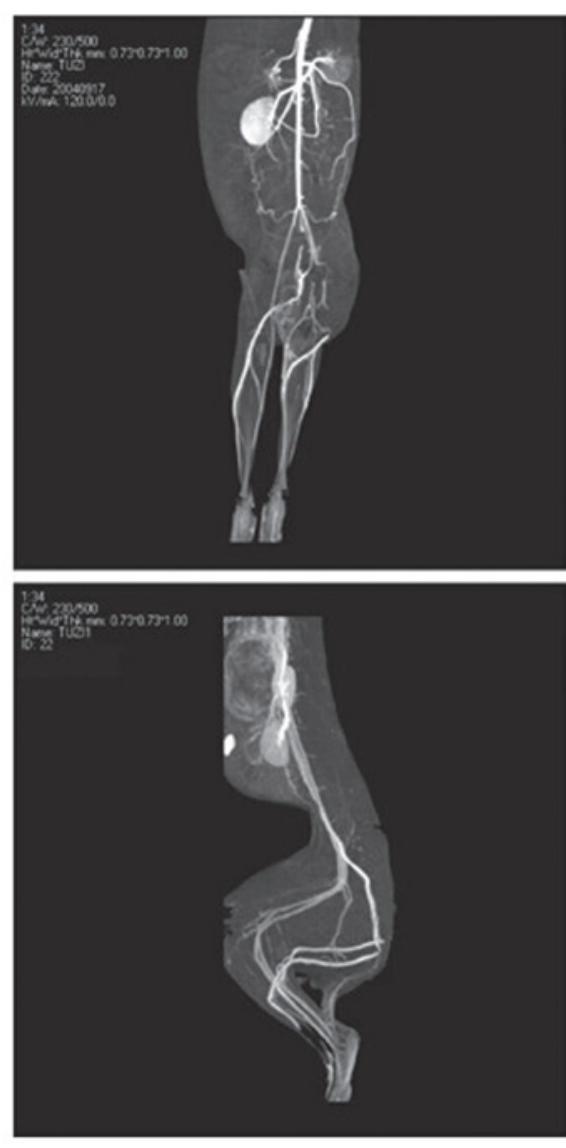

B
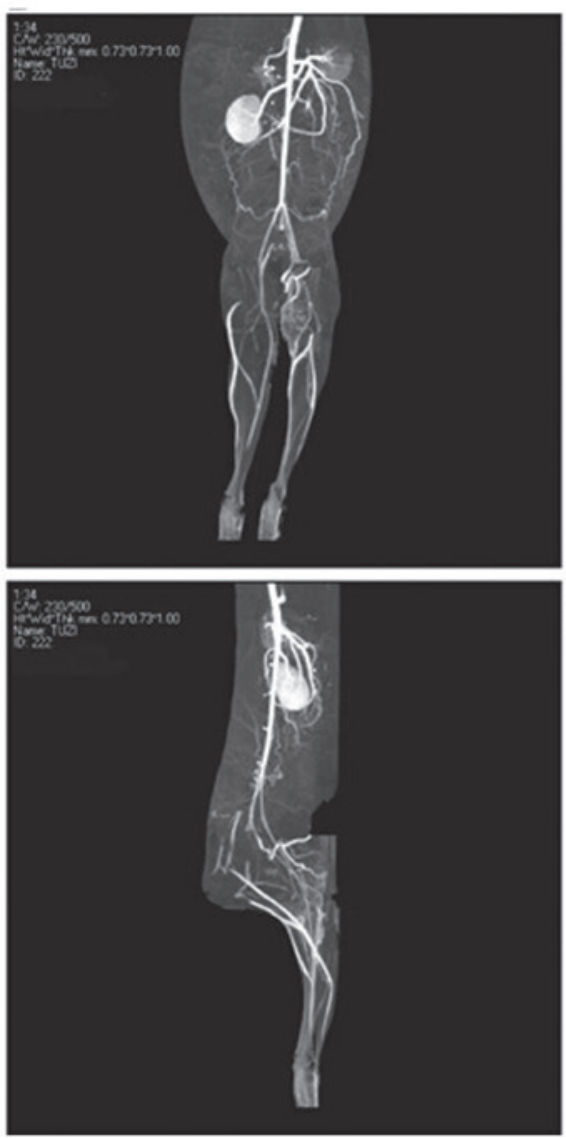

C
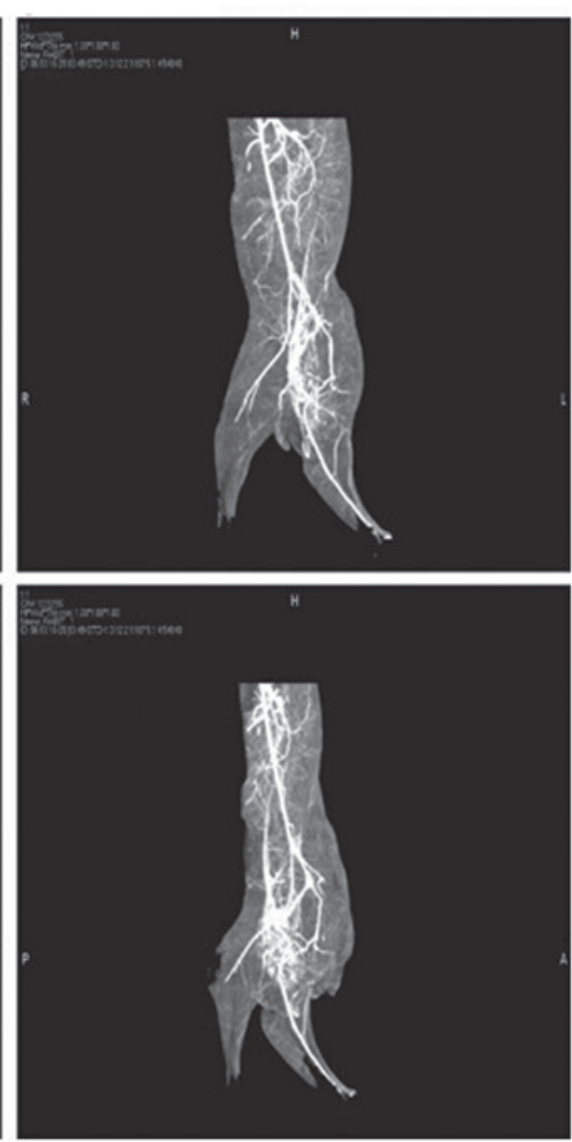

Figure 9. Analysis of recanalization by computerizezd tomography ateriography (CTA). (A) The control group was treated with culture medium. (B) Ad-transfected EPCs (Ad/EPCs) transplantation group; (C) Ad-VEGF165-transfected EPCs (Ad-VEGF/EPCs) transplantation group.

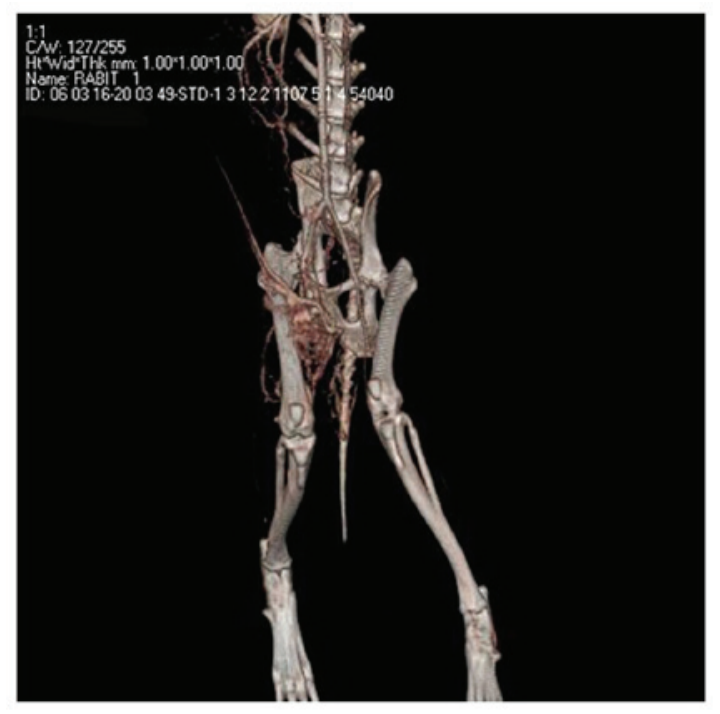

Figure 10. Analysis of the establishment of collateral circulation in group C was observed following soft tissue removal.

In the present study, the Brdu-labeled EPCs were transplanted into New Zealand rabbits, and seven days later muscle tissue specimens of the ischemic limbs were obtained. The results of immunohistochemical staining demonstrated that the labeled cells had incorporated into the blood vessel wall.
Kocher et al (14) also observed that transplanted EPCs were able to directionally integrate into the new blood vessels of ischemic limbs. In addition, previous studies demonstrated that the mRNA expression levels of VEGF in ischemic muscles were significantly higher, as compared with non-ischemic muscles $(15,16)$. Therefore, these results suggest that the predominant cause of EPC integration into the most severe ischemic muscle area were the elevated expression levels of VEGF in the ischemic area.

EPCs were transplanted into New Zealand rabbits following limb ischemic experimentation, and the muscle tissue specimens of ischemic limbs were obtained and analyzed by immunohistochemical staining and CTA. As compared with the control and Ad-GFP/EPCs groups, there was a greater density of new blood vessels in the ischemic area, and collateral circulation was greater in the Ad-GFP-VEGF165/EPCs group.

EPCs are easy to acquire, non-immunogenic so that they do not cause immune rejection, and are able to migrate to the area of new blood vessels. In addition, the proteins released by EPCs readily enter the bloodstream and migrate throughout the body where they have extensive roles. Therefore, EPCs may prove to be a successful target for gene therapy.

Numerous studies have investigated the effects of VEGF on angiogenesis $(17,18)$. In the present study, concentration levels of VEGF in the supernatant of the Ad-GFP-VEGF165/EPCs were markedly higher, as compared with those in the Ad-GFP/EPCs and non-Ad/EPCs. The results of the present study suggest that 
transplantation with VEGF-transduced EPCs may improve the function of EPCs, and may also have a role in angiogenesis via the action of exocrine proteins. The present study also provides the basis for the use of EPC transplantation in the treatment of limb ischemia.

\section{References}

1. Suzuki H, Shibata R, Kito T, Ishii M, Li P, Yoshikai T, Nishio N, Ito $\mathrm{S}$, Numaguchi, Yamashita JK, et al: Therapeutic angiogenesis by transplantation of induced pluripotent stem cell-derived Flk-1 positive cells. BMC Cell Biol 11: 72, 2010.

2. Brenes RA, Jadlowiec CC, Bear M, Hashim P, Protack CD, Li X, Lv W, Collins MJ and Dardik A. Toward a mouse model of hind limb ischemia to test therapeutic angiogenesis. J Vasc Surg 56 $1669-1679,2012$

3. Kang HC, Kim DS, Kim JY, Kim HS, Lim BY, Kim HD, Lee JS, Eun BL and Kim DW: Behavioral improvement after transplantation of neural precursors derived from embryonic stem cells into the globally ischemic brain of adolescent rats. Brain Dev 32: 658-668, 2010.

4. Zhou Y, Singh AK, Hoyt RF Jr, Wang S, Yu Z, Hunt T, Kindzelski B, Corcoran PC, Mohiuddin MM and Horvath KA: Regulatory T cells enhance mesenchymal stem cell survival and proliferation following autologous co-transplantation in ischemic myocardium. J Thorac Cardiovasc Surg 148: 1131-1137, 2014.

5. Guzmán-Hernández ML, Potter G, Egervári K, Kiss JZ and Balla T: Secretion of VEGF-165 has unique characteristics, including shedding from the plasma membrane. Mol Biol Cell 25: 1061-1072, 2014

6. Huang J, Chen J, Wang W, et al: Birthdate study of GABAergic neurons in the lumbar spinal cord of the glutamic acid decarboxylase 67-green fluorescent protein knock-in mouse. Front Neuroanat 7: 42, 2013.

7. Fu SS, Li FJ, Wang YY, You AB, Qie YL, Meng X, Li JR, Li BC, Xhang Y and Da Li Q: Kallikrein gene-modified EPCs induce angiogenesis in rats with ischemic hindlimb and correlate with integrin $\alpha v \beta 3$ expression. PLoS One 8: e73035, 2013.

8. Ahn GO and Brown M: Role of endothelial progenitors and other bone marrow-derived cells in the development of the tumor vasculature. Angiogenesis 12: 159-164, 2009.
9. Otani A, Kinder K, Ewalt K, Otero FJ, Schimmel P and Friedlander M: Bone marrow-derived stem cells target retinal astrocytes and can promote or inhibit retinal angiogenesis. Nat Med 8: 1004-1010, 2002.

10. Smith C and Storms B: Hematopoietic stem cells. Clin Orthop Relat Res 379 (Suppl): S91-S97, 2000.

11. Khakoo AY and Finkel T: Endothelial progenitor cells. Annu Rev Med 56: 79-101, 2005.

12. Hristov M, Erl W and Weber PC: Endothelial progenitor cells: Isolation and characterization. Trends Cardiovasc Med 13: 201-206, 2003.

13. Asahara T, Murohara T, Sullivan A, Silver M, van der Zee R, Li T, Witzenbichler B, Schatteman G and Isner JM: Isolation of putative progenitor endothelial cells for angiogenesis. Science 275: 964-967, 1997.

14. Kocher AA, Schuster MD, Szaboles MJ, Takuma S, Burkhoff D, Wang J, Homma S, Edwards NM and Itescu S: Neovascularization of ischemic myocardium by human bone-marrow-derived angioblasts prevents cardiomyocyte apoptosis, reduces remodeling and improves cardiac function. Nat Med 7: 430-436, 2001.

15. Sellke FW, Wang SY, Stamler A, Lopez JJ, Li J, Li J and Simons M: Enhanced microvascular relaxations to VEGF and bFGF in chronically ischemic porcine myocardium. Am J Physiol 271: H713-H720, 1996.

16. Nakano M, Satoh K, Fukumoto Y, Ito Y, Kagaya Y, Ishii N, Sugamura K and Shimokawa H: Important role of erythropoietin receptor to promote VEGF expression and angiogenesis in peripheral ischemia in mice. Circ Res 100: 662-669, 2007.

17. Wolff T, Mujagic E, Gianni-Barrera R, Fueglistaler P, Helmrich U, Misteli H, Gurke L, Heberer M and Banfi A. FACSpurified myoblasts producing controlled VEGF levels induce safe and stable angiogenesis in chronic hind limb ischemia. J Cell Mol Med 16: 107-117, 2012.

18. Mujagic E, Gianni-Barrera R, Trani M, Patel A, Gürke L, Heberer M, Wolff T and Banfi A: Induction of aberrant vascular growth, but not of normal angiogenesis, by cell-based expression of different doses of human and mouse VEGF is speciesdependent. Hum Gene Ther Methods 24: 28-37, 2013. 